\title{
Dermatite digital bovina: resposta terapêutica e custo dos protocolos adotados em duas propriedades rurais
}

\section{Cattle digital dermatitis: response and economic analysis to therapeutic protocols adopted in two farms}

\author{
Maria Auxiliadora Leão, ${ }^{*}$ Maria Clorinda Soares Fioravanti, ${ }^{*}$ Olízio Claudino Silva, ${ }^{*}$ José Serafim, ${ }^{*}$ Maria Ivete Moura, ${ }^{*}$ \\ Leandro Batista Caetano, ${ }^{*}$ Duvaldo Eurides, ${ }^{* *}$ Luiz Antônio Franco Silva*
}

\begin{abstract}
Resumo
Este trabalho avaliou a evolução e os custos do tratamento de lesões de dermatite digital, em bovinos da raça Girolando submetidos a diferentes protocolos terapêuticos, empregando 42 fêmeas portadoras da enfermidade, em duas propriedades rurais. Em cada propriedade, os animais foram distribuídos em três grupos (GI, GIl, e GIII) de sete animais cada, sendo 21 oriundos da propriedade A e os demais da propriedade B. Os animais do Gl receberam antibioticoterapia parenteral, as feridas cirúrgicas tratadas com medicamento local e, após sete dias, passagem em pedilúvio contendo solução de sulfato de cobre a $3 \%$, intercalando-se, semanalmente, com solução de hipoclorito de sódio a 1\%. Nos animais de Gll as feridas cirúrgicas foram apenas protegidas com algodão ortopédico e ataduras de crepom. A partir do sétimo dia da intervenção cirúrgica, foram submetidos ao mesmo manejo do Gl. Nos bovinos do grupo GIII (controle), sete dias após tratamento cirúrgico, procedeu-se à retirada da atadura e passagem em pedilúvio contendo apenas água. Os animais foram avaliados com 7, 15, 30,45 e 60 dias do pós-operatório, utilizando escores clínicos de cicatrização como parâmetros da evolução clínica das feridas cirúrgicas. Estimou-se os custos dos procedimentos baseando-se no material de consumo e mão-de-obra, entre outros. Observou-se maior número de animais recuperados no $\mathrm{Gl}$, mas não houve diferença estatística $(p>0,05)$ quando comparados aos animais do GII, sendo os protocolos terapêuticos I e II apresentando a mesma eficácia. Apenas o tratamento cirúrgico não foi suficiente para recuperar os animais do GIll. O custo estimado, em dólares, por animal foi de US\$ 69,41 para o tratamento GI, US\$ 54,02 para o Gll e US\$ 51,96 para o GIII.
\end{abstract}

Palavras-chave: casco, custos, doença digital, tratamento.

\begin{abstract}
The scope of this study was to evaluate the response of early phase digital dermatitis wounds to different therapeutic protocols, in Gir breed cattle in two distinct farms subjected to the same management, and also to estimate the costs of the treatments proposed. The study was conducted simultaneously during the period of april 2004 and april 2005, in 42 female cattle bearing wounds clinically characteristic of early phase digital dermatitis. The animals were allocated in three groups (GI, GII, GIII) of seven animals, where 21 were from farm A, and the remaining from farm B. Animals of group one (GI) received parenteral antibiotic therapy, and their surgical wounds were treated with a local treatment protocol, and when the bandage was removed, on the seventh day, they passed in a footbath with three-percent copper solution, weekly and intercalated to a one-percent sodium hypochlorite solution. Animals from group two (GII) did not receive local or parenteral antibiotic therapy, having their surgical wounds protected with orthopedic cotton and bandage right after curettage, then being subjected to the same management of GI. The animals of group three (GIII) constituted the control group, therefore receiving neither local nor parenteral antibiotic therapy, and after the seventh day, after removing the bandage, passed in a footbath water only. All animals were evaluated considering clinical scores of healing associated to clinical parameters of surgical wound evolution, at days $7,15,30$, 45 and 60 counting from the surgical day. Procedures costs were estimated based upon consumption material and human labor among other costs. Treatment GI healed a greater number of animals, but there was no statistic difference $(p>0,05)$ when compared to surgical treatment associated to footbath (GII). Surgical treatment alone was not enough to recover a significant amount of wounds, therefore not recommended as a treatment for early phase digital dermatitis. Therapeutic protocols I and II had the same efficiency, no matter the season of the year, but the healing scores were influenced by therapeutic protocols. The estimated costs per treatment were of US\$ 69,41 for GI, US\$ 54,02 for GII and US\$51,96 for GIII.
\end{abstract}

Keywords: bovine, hoof, treatment, costs.

* Escola de Veterinária da Universidade Federal de Goiás, Estrada saída Goiânia-Nova Veneza Km 0, Setor Samambaia, Campus II. Cx. Postal: 131. CEP: 74001-970. lafranco@vet.ufg.br

** Universidade Federal de Uberlândia, Instituto de Ciências Biomédicas, Instituto de Medicina Veterinária. Av. Pará, 1720 - Bloco 2 T Umuarama CEP: 38400-902- Uberlândia, MG - Brasil. duvaldo@ufu.br 


\section{Introdução}

As lesões de dermatite digital bovina são freqüentes na face plantar do casco próxima à margem coronária e na comissura entre os bulbos dos talões, envolvendo predominantemente a camada epidérmica e em menor extensão a derme (Nicoletti, 2004). Segundo Bergsten (1997) e Greenough (1997), a lesão na fase inicial da doença caracteriza-se por inflamação interdigital altamente infecciosa, seguida por ulceração na epiderme, próxima à zona de crescimento do casco, podendo evoluir para a forma erosiva. Nicoletti (2004) afirmou que a lesão é circunscrita por uma borda epitelial branca, com centro avermelhado e inúmeras papilas córneas brancas, conferindo à ferida um aspecto de morango.

A dermatite digital tem recebido destaque especial em função da distribuição mundial, da alta prevalência e rápida disseminação nos rebanhos, além das dificuldades de controle (Luginbuhl e Kollbrunner, 2000). Segundo Nicoletti (2004), a doença está disseminada principalmente entre os rebanhos leiteiros em várias regiões do país. Silva et al. (2001a), estudando as características clínicas e epidemiológicas das enfermidades podais na bacia leiteira de OrizonaGO, observaram freqüência de $24,36 \%$ de dermatite digital, sendo $14,53 \%$ de dermatite digital verrucosa. Marega (2001) afirmou que a ocorrência de dermatite digital em rebanhos brasileiros mostrou as mesmas tendências mundiais, com prevalência de $5,7 \%$ para rebanhos leiteiros e $5,2 \%$ para gado de corte.

O diagnóstico da dermatite digital é realizado com base no histórico clínico do início de uma epidemia, em que os animais apresentam desconforto e claudicação em animais do rebanho (Bergsten, 1997). Para Cunha (2000), Marega (2001), Silva et al. (2001b), Manske et al. (2002) e Ferreira (2003), recomenda-se no tratamento da dermatite digital a intervenção cirúrgica, antibioticoterapia local, parenteral ou em pedilúvios. Greenough e Weaver, (1997) recomendaram o uso de bandagens nos casos onde é realizada intervenção cirúrgica e Ferreira (2003) indicou o uso de tamancos de madeira (ipê) fixados com resina acrílica na sola do dígito saudável. Kamiloglo et al. (2002) concluíram que a resposta ao tratamento da dermatite digital bovina com aplicação local de oxitetraciclina é lenta, porém eficiente, quando realizadas quatro aplicações na fase inicial do processo. Segundo Nicoletti (2004), o pedilúvio contendo solução antibiótica para tratamento da dermatite digital é bastante discutível, uma vez que é onerosos e, se for realizado tardiamente, torna-se ineficiente.

Para o controle da enfermidade faz-se necessário, além do tratamento medicamentoso, adotar medidas como higiene das instalações, exames periódicos no rebanho, quarentena de animais introduzidos na propriedade, diminuição da densidade animal nos lotes, uso correto do pedilúvio, redução da distância percorrida dos piquetes até a sala de ordenha e esterilização do material de casqueamento (Bergsten, 1997; Dias e Marques Júnior, 2003; Nicoletti, 2004).

Este trabalho objetivou avaliar a resposta de lesões de dermatite digital em sua fase inicial a diferentes protocolos terapêuticos em bovinos Girolandos, submetidos ao mesmo manejo, em duas propriedades rurais, bem como os custos dos diferentes tratamentos estabelecidos.

\section{Material e métodos}

O estudo foi realizado, simultaneamente, em duas propriedades rurais, identificadas como $\mathrm{A}$ e $\mathrm{B}$, localizadas na região Centro-Sul do Estado de Goiás, distantes aproximadamente 500 quilômetros uma da outra e destinadas à criação extensiva de bovinos de aptidão leiteira. Cada fazenda contava, na ocasião, com um efetivo fixo de aproximadamente 1.000 animais. As fêmeas em lactação eram manejadas extensivamente em pastagens de Brachiaria decumbens no período chuvoso. No período seco do ano eram mantidas confinadas e suplementadas com cana-de-açúcar triturada, uréia e concentrado mineral e protéico, em dois fornecimentos diários. A formulação e a quantidade oferecida do suplemento variavam de acordo com a produção individual.

A pesquisa foi realizada entre abril de 2004 e abril de 2005, utilizando-se 42 fêmeas bovinas, com idade entre três e nove anos, portadoras de lesões clínicas características de dermatite digital conforme descritas por Nicoletti (2004), sendo 21 oriundas da propriedade A e as demais da B. Em cada propriedade os bovinos foram distribuídos de acordo com o protocolo terapêutico, em três grupos compostos por sete animais cada, denominados de Gl, Gll e GIII ou protocolos I, II e III. Para facilitar a observação das lesões, removeram-se sujidades e crostas das feridas e realizou-se exame podológico específico, seguindo as recomendações de Dirksen (1993). Após o diagnóstico inclui-se na pesquisa apenas bovinos com lesões no espaço interdigital, entre os talões e odor acre característico da doença, presença de secreção serosa, aspecto erosivo e hiperemia com alguns pontos esbranquiçados, presença de pêlos e moderada sensibilidade à palpação (Figura 1), sendo todos os casos considerados na fase inicial da doença, com base nas descrições de Greenough e Weaver (1997) e Nicoletti (2004).

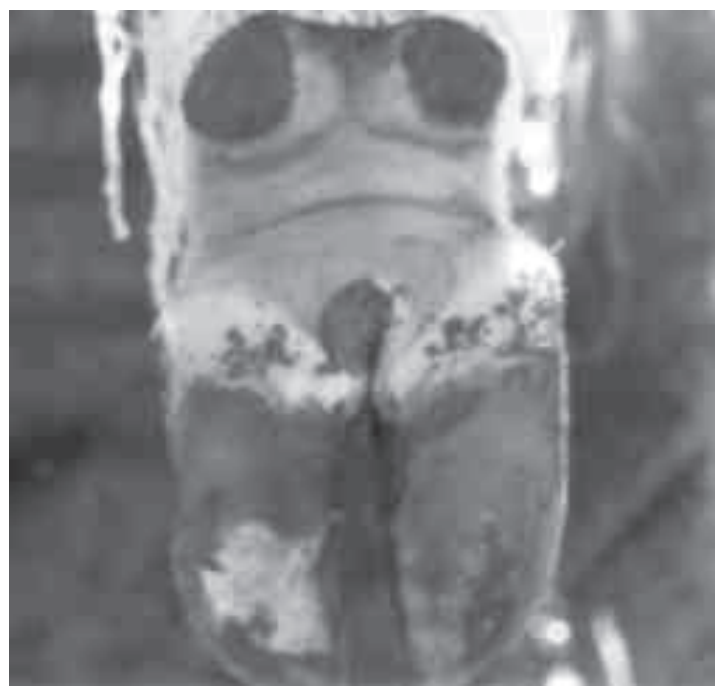

Figura 1: Dermatite digital bovina - fase inicial - hiperemia e erosão no espaço interdigital entre os talões

O pré-operatório constou de jejum hídrico e alimentar de no mínimo 12 horas, tranqüilização com cloridrato de xilazina a 2\% (Anasedanâ, Sespo Indústria e Comércio Ltda. - Divisão Vetbrands Saúde Animal - Jacareí-SP), na dose de 0,05 a 0,1 $\mathrm{mg} / \mathrm{kg}$ de peso corporal, via intravenosa (Massone, 2003), e contenção em decúbito dorsal ou em bretes apropriados, 
limpeza e higienização das feridas com água, sabão e iodophor (BiocidâLaboratórios Pfizer Ltda., Guarulhos, SP). Empregou-se na anestesia intravenosa (Massone, 2003) pelo método de Bier, cloridrato de lidocaína a $2 \%$ (Dorfinâ, Laboratório Hertape, Juatuba, MG).

A curetagem das feridas foi realizada em todos os animais, independentemente da propriedade e do grupo ao qual pertenciam. A realização do tratamento cirúrgico das lesões e do toalete dos estojos córneos comprometidos e saudáveis foi conduzida conforme descrito por Cunha (2000). Nos bovinos pertencentes ao $\mathrm{Gl}$, aplicou-se sobre as feridas cirúrgicas solução contendo percloreto de ferro, iodo metálico e salicilato de metila (Hemosthalâ - Minerthal Produtos Agropecuários Ltda., SP) e oxitetraciclina pó (Terramicina pó solúvel com Antigerm $77 \AA$ - Laboratório Pfizer Ltda. - Divisão de Saúde Animal - Guarulhos, SP). Concomitantemente empregou-se oxitetraciclina, OxitapeLA ${ }^{\circledR}$ - Laboratório Hertape - Juatuba, MG). Este grupo na dose de $20 \mathrm{mg} / \mathrm{kg}$ de peso corporal, via intramuscular, em intervalos de 48 horas, até completar três aplicações. As lesões, após serem tratadas, foram protegidas com algodão hidrófilo e sobre este foi pulverizado sulfato de cobre. Seqüencialmente, as feridas foram envolvidas por ataduras de crepom (Ortho Surgical Superintendência Hospitalar Ltda., Santa Bárbara, SP) e impermeabilizadas com produto à base de diclorodivinilpivolidona, orto-ortodimetil para-nitrofenil-fosforodioato e alcatrão vegetal esterilizado (Miosthal ${ }^{\circledR}$ - Minerthal Produtos Agropecuários Ltda., São Paulo, SP).

Nos animais alocados nos grupos, Gll e GIII, após tratamento cirúrgico das lesões e toalete dos cascos, protegeram-se as feridas cirúrgicas com algodão hidrófilo e ataduras de crepom, porém sem aplicação local e parenteral de medicamentos. Sete dias após o procedimento cirúrgico, a bandagem protetora foi retirada e os bovinos pertencentes ao GIII foram conduzidos, diariamente, a um pedilúvio contendo apenas água trocada a cada 48 horas, por um período de 60 dias. Nos bovinos pertencentes aos grupos Gl e Gll, após a remoção da bandagem protetora, as feridas cirúrgicas foram tratadas por um período de 60 dias, em pedilúvio contendo solução de sulfato de cobre a $3 \%$ ou hipoclorito de sódio a $1 \%$, de acordo com o preconizado por Silva et al. (2005), obedecendo-se a um intervalo de 48 horas para efetuar as trocas das soluções. Estipulou-se que o rodízio entre as soluções ocorreria semanalmente por um período de dois meses.

Para se avaliar a eficácia do protocolo terapêutico foram estabelecidos escores de cicatrização definidos no Quadro 1.

\begin{tabular}{|c|c|}
\hline Escores & Porcentagem de cicatrização \\
\hline Escore 0 & Ausência \\
\hline Escore 1 & Até $25 \%$ \\
\hline Escore 2 & $26 \%$ a $50 \%$ \\
\hline Escore 3 & $51 \%$ a $75 \%$ \\
\hline Escore 4 & $76 \%$ a $100 \%$ \\
\hline
\end{tabular}

Quadro 1: Escores de cicatrização para avaliação clínicas durante o período pós-operatório dos animais experimentais portadores de dermatite digital

As dimensões das lesões foram medidas nos sentidos longitudinal e transversal logo após o tratamento cirúrgico (momento zero - M0), aos sete dias (M7) e, subseqüentemente, com intervalos de 15 dias até completar 60 dias (M15, M30, M45 e M60), quando as avaliações foram encerradas.
Consideraram-se como recuperados apenas os bovinos que apresentaram escore 4 ao final das avaliações. Os custos dos protocolos terapêuticos empregados foram estimados, considerando-se o material de consumo utilizado no pré, trans e pós-operatório, os honorários de um veterinário autônomo e a construção de pedilúvio.

Para avaliar o efeito do manejo da propriedade rural, do tratamento e períodos do ano sobre os animais recuperados e não recuperados, utilizou-se a análise do desvio (Anodev), segundo Cordeiro (1986). Para avaliar o processo cicatricial foi realizada a transformação matemática do escore, de modo que nos testes estatísticos usou-se a raiz quadrada dos escores. $\mathrm{Na}$ análise estatística da variável escore, os dados foram transformados e as médias comparadas pelo Teste de Tukey (5\%), utilizando-se o "General Linear Models Procedure" do "Statistical Analysis System" SAS (1997).

\section{Resultados e discussão}

O exame clínico realizado de acordo com as recomendações de Dirksen (1993) e Greenough (2000) e a contenção dos animais em bretes apropriados possibilitou a remoção das crostas e sujidades, permitiu a conclusão do diagnóstico e a realização das intervenções cirúrgicas, proporcionando segurança ao cirurgião e auxiliar, dispensando o jejum e o uso de tranqüilizantes, portanto, minimizando os prejuízos devido à redução na produção de leite nos dias subseqüentes ao tratamento cirúrgico. Para Shearer (1998), os diagnósticos precoces associados aos tratamentos apropriados para doenças podais minimizam as perdas econômicas e reduzem o sofrimento do animal, além de serem mais eficazes.

Na propriedade A, 60 dias após o início do tratamento, observou-se recuperação de sete animais (100\%) submetidos ao protocolo terapêutico I, seis animais $(85,72 \%)$ do protocolo II e dois animais $(28,57 \%)$ do protocolo terapêutico III. Os mesmos procedimentos terapêuticos quando empregados nos bovinos da propriedade $B$ resultaram na cura de cinco animais $(71,43 \%)$ submetidos ao protocolo I, quatro animais $(57,14 \%)$ ao protocolo II e dois animais $(28,57 \%)$ ao protocolo III (Tabela 1).

Tabela 1: Distribuição de bovinos Girolandos, de acordo com o protocolo terapêutico e índice de recuperação após tratamento da dermatite digital, em duas propriedades rurais do Estado de Goiás, durante os meses de abril de 2004 a abril de 2005

\begin{tabular}{c|c|c|c}
\hline Grupo/Propriedade & \multicolumn{2}{|c|}{ Resultados dos tratamentos } & \multirow{2}{*}{ Total } \\
\cline { 2 - 3 } & Recuperados & Não recuperados & \\
\hline GI-A & $7(100 \%)$ & $0(0 \%)$ & 7 \\
\hline GII-A & $6(85,72 \%)$ & $1(14,28 \%)$ & 7 \\
\hline GIII-A & $2(28,57 \%)$ & $5(71,43 \%)$ & 7 \\
\hline GI-B & $5(71,43 \%)$ & $2(28,57 \%)$ & 7 \\
\hline GII-B & $4(57,14 \%)$ & $3(42,86 \%)$ & 7 \\
\hline GIII-B & $2(28,57 \%)$ & $5(71,43 \%)$ & 7 \\
\hline TOTAL & 26 & 16 & 42 \\
\hline
\end{tabular}

GI (Grupo I): Curetagem, tratamento local, parenteral e pedilúvio; GI (Grupo II): Curetagem e pedilúvio; GIII (Grupo III): Curetagem e pedilúvio com água (controle) 
Não se observou diferença significativa entre os protocolos terapêuticos I e II nas duas propriedades avaliadas. Esta uniformidade de resposta pode ser atribuída, em parte, à padronização do rebanho, manejo, infra-estrutura física e à mão-de-obra auxiliar qualificada. Entretanto, foi observada diferença significativa quando se comparou o protocolo terapêutico I com o III e II com o III (Tabela 2), mostrando que apenas o tratamento cirúrgico não foi suficiente para promover a cura de um número significativo de lesões. Neste contexto, é possível inferir que após a curetagem cirúrgica das lesões é necessário promover o tratamento local da ferida empregando antibióticos, local e parenteral, bem como o tratamento em pedilúvio, conduta que encontra respaldo nos trabalhos de Cunha (2000) e Manske et al. (2002). Diante desses achados, infere-se que o pedilúvio contendo apenas água, além de não promover a cura das lesões em sua plenitude, pode ser uma fonte de transmissão da dermatite digital, reforçando a importância do emprego de soluções sanitizantes ou antibióticas quando se utiliza esse método para proceder ao tratamento local de feridas digitais dos bovinos.

Diante desta constatação, embora numericamente o protocolo terapêutico II tenha recuperado menor número de bovinos do que o protocolo I, não existe justificativa econômica para empregar antibioticoterapia local e parenteral no tratamento de lesões iniciais da dermatite digital quando se fizer uso de soluções de sulfato de cobre e/ou hipoclorito de sódio em pedilúvio.

Tabela 2: Efeito do protocolo terapêutico (grupo) e período do ano (seca e chuva) sobre o resultado do tratamento da dermatite digital de bovinos em duas propriedades rurais do Estado de Goiás, durante os meses de abril de 2004 a abril de 2005

\begin{tabular}{|c|c|c|c|}
\hline Efeito & \multicolumn{2}{|c|}{ Grupo } & Nível de significância \\
\hline \multirow{3}{*}{ Grupo } & $\mathrm{I}$ & II & $p>0,1022$ \\
\hline & I & III & $P<0,0015$ \\
\hline & III & "III" & $P<0,0128$ \\
\hline Efeito & Período & & Nível de significância \\
\hline Período & Chuva & Seca & $p>0,3851$ \\
\hline
\end{tabular}

GI (Grupo I): Curetagem, tratamento local, parenteral e pedilúvio; GII (Grupo II): Curetagem e pedilúvio; GIII (Grupo III): Curetagem e pedilúvio com água (Controle), Seca - período compreendido entre maio e setembro, Chuva - período compreendido entre outubro e abril

Ao avaliar-se o efeito do período do ano sobre a recuperação dos animais, não foi observada diferença significativa entre os dois criatórios (Tabela 2). Este resultado mostra que os protocolos terapêuticos adotados neste estudo apresentaram a mesma eficácia, independentemente da estação do ano em que os animais foram tratados. Na literatura consultada (Manske et al., 2002; Silva et al., 2005) não foram encontradas referências sobre o índice de recuperação de bovinos portadores de dermatite digital submetidos a diferentes protocolos terapêuticos, simultaneamente em mais de uma propriedade rural.

No período seco, dez (47,63\%) bovinos se recuperaram na propriedade A, enquanto na propriedade B ocorreu a cura de quatro $(19,06 \%)$ animais (Tabela 3$)$. Ressalte-se que nessa ocasião, na propriedade B ocorreram substituições não programadas da mão-de-obra auxiliar, demandando alguns meses para que as pessoas contratadas recebessem 0 treinamento necessário para conduzir adequadamente o pósoperatório. Diante desta situação, fica claro que o fator mãode-obra é de grande importância para a condução do pósoperatório. Girolando, clinicamente portadores de dermatite digital e submetidos a três tratamentos no pós-operatório, no período seco e chuvoso, em duas propriedades rurais do Estado de Goiás, durante os meses de abril de 2004 a abril de 2005

\begin{tabular}{l|l|l|l|l|l}
\hline \multirow{2}{*}{ Propriedades } & \multicolumn{4}{|c|}{ Número de animais tratados } & \multirow{2}{*}{ Total } \\
\cline { 2 - 5 } & \multicolumn{2}{|c|}{ Período seco (intensivo) } & Período chuvoso (extensivo) & \\
\hline & Recuperados & $\begin{array}{c}\text { Não } \\
\text { recuperados }\end{array}$ & Recuperados & $\begin{array}{c}\text { Não } \\
\text { recuperados }\end{array}$ & \\
\hline A & $10(47,63 \%)$ & $3(14,28 \%)$ & $5(23,81 \%)$ & $3(14,28 \%)$ & 21 \\
\hline B & $4(19,06 \%)$ & $7(33.33 \%)$ & $7(33,33 \%)$ & $3(14,28 \%)$ & 21 \\
\hline Total & $\mathbf{1 3}$ & $\mathbf{1 0}$ & $\mathbf{1 2}$ & $\mathbf{7}$ & $\mathbf{4 2}$ \\
\hline
\end{tabular}

GI (Grupo I): Curetagem, tratamento local, parenteral e pedilúvio; GII (Grupo II): Curetagem, local e parenteral, pedilúvio com água (controle); Fazenda A - Jataí (GO); Fazenda B Orizona (GO); Seca - período compreendido entre maio e setembro; Chuva - período compreendido entre outubro e abril.

A avaliação da interação entre os efeitos fazenda e período do ano na propriedade $A$ mostrou que não houve diferença significativa $(p>0,05)$ no resultado dos tratamentos entre os períodos seco e chuvoso. Porém, na propriedade B o período do ano exerceu influência na recuperação dos bovinos, sendo que sete $(33,33 \%)$ animais se recuperaram no período chuvoso e somente quatro $(19,06 \%)$ no período seco (Tabela 3). Nessa propriedade, o solo pedregoso pode ter propiciado menor retenção de umidade, aspecto que Silva et al. (2001a) refutaram como importante na recuperação de bovinos portadores de lesões digitais.

Os bovinos pertencentes ao grupo GIII apresentaram os piores escores de cicatrização (Tabela 4), tanto na propriedade A como na B. Este achado reforça o fato de que somente o procedimento cirúrgico não foi suficiente para debelar o processo infeccioso local, sendo necessária a adoção de outras medidas auxiliares, como o emprego local de soluções medicamentosas.

Comparando os escores de cicatrização obtidos empregando os protocolos terapêuticos I e II, verifica-se que não se observou diferença significativa ( $p>0 ; 05)$, indicando que a evolução clínica do processo cicatricial apresentou o mesmo comportamento quando foram usados os dois tratamentos ao mesmo tempo. Todavia ao comparar, em ambas as propriedades, os protocolos I com o III e II com o III, observouse diferença significativa $(p<0,05)$ (Tabela 5$)$. 
Tabela 4: Escores clínicos de cicatrização das lesões de dermatite digital bovina em diferentes momentos de avaliação, após o tratamento realizado no período de abril de 2004 a abril de 2005 em duas propriedades rurais do Estado de Goiás

\begin{tabular}{|c|c|c|c|c|c|c|}
\hline \multirow{2}{*}{\multicolumn{2}{|c|}{$\begin{array}{l}\text { Grupo / } \\
\text { Fazenda }\end{array}$}} & \multicolumn{5}{|c|}{ Momentos de avaliação (dias) } \\
\hline & & 7 dias & 15 dias & 30 dias & 45 dias & 60 dias \\
\hline \multirow{2}{*}{ I } & $\mathrm{A}$ & $1,24^{*}\left(1,57^{* *}\right)$ & $1,63(2,71)$ & $2,10(4,43)$ & $2,20(4,86)$ & $2,36(5,00)$ \\
\hline & $\mathrm{B}$ & $1,18(1,43)$ & $1,48(2,29)$ & $1,91(3,71)$ & $1,86(3,57)$ & $2,05(4,28)$ \\
\hline \multirow[b]{2}{*}{ II } & A & $1,06(1,14)$ & $1,40(2,00)$ & $1,72(3,00)$ & $2,07(4,28)$ & $2,16(4,71)$ \\
\hline & $B$ & $1,12(1,28)$ & $1,28(1,71)$ & $1,67(2,86)$ & $1,94(3,86)$ & $2,01(4,14)$ \\
\hline \multirow{2}{*}{ III } & A & $1,00(1,00)$ & $1,06(1,14)$ & $1,10(1,28)$ & $1,20(1,57)$ & $1,34(2,00)$ \\
\hline & $B$ & $1,00(1,00)$ & $1,06(1,14)$ & $1,16(1,43)$ & $1,31(1,86)$ & $1,47(2,43)$ \\
\hline
\end{tabular}

*Raiz quadrada do escore ** Média do escore; GI (Grupo I): Curetagem, tratamento local, parenteral e pedilúvio; GII (Grupo II): Curetagem e pedilúvio; GIII (Grupo III): Curetagem e pedilúvio com água (controle); Fazenda A - Jataí (GO), Fazenda B - Orizona (GO)
Entre os três tratamentos testados, em um período de 60 dias os dados revelaram que houve diferença significativa $(p<0,05)$ quanto à cicatrização de lesões de dermatite digital bovina nas propriedades A e B. Na propriedade A, o tratamento testado para os bovinos alocados no grupo Gl mostrou desempenho superior aos outros tratamentos e no intervalo entre 45 e 60 dias de observação, os animais apresentaram os melhores escores de cicatrização. Os tratamentos I e II mostraram índices de cicatrização muito semelhantes a partir de 45 dias (Tabela 6). Silva et al. (2005) após realizar a curetagem cirúrgica, aplicação tópica e parenteral de medicamentos, recuperaram

Tabela 5: Resultados das médias dos escores de cicatrização de acordo com o grupo avaliado e a fazenda avaliada

\begin{tabular}{ccccc}
\hline \multicolumn{2}{c}{ Fazenda A (Jataí-Go) } & \multicolumn{2}{c}{ Fazenda B (Orizona-Go) } \\
\hline Grupo & Média & Escore & Média & Escore \\
\hline I & $1,88^{\mathrm{A}}$ & $1-4$ & $1,69 \mathrm{~A}$ & $1-4$ \\
\hline II & $1,68^{\mathrm{A}}$ & $1-4$ & $1,61 \mathrm{~A}$ & $1-4$ \\
\hline III & $1,14 \mathrm{~B}$ & $1-4$ & $1,20 \mathrm{~B}$ & $1-4$ \\
\hline
\end{tabular}

*Letras iguais na mesma coluna indicam médias equivalentes; **nível de significância 0,05; GI (Grupo I): Curetagem, tratamento local, parenteral e pedilúvio; GII (Grupo II): Curetagem com pedilúvio; GIII (Grupo III): Curetagem, pedilúvio com água (controle)

Tabela 6: Resultado das médias dos escores de cicatrização ao longo do tempo de acordo com o período e a Fazenda avaliada

\begin{tabular}{ccc}
\hline Período & Fazenda A & Fazenda B \\
\cline { 2 - 3 } & Média & Média \\
\hline M60 & $3,90 \mathrm{~A}$ & $3,62^{\mathrm{a}}$ \\
\hline M45 & $3,57 \mathrm{~A}$ & $3,10 \mathrm{AB}$ \\
\hline M30 & $2,90 \mathrm{~B}$ & $2,67 \mathrm{~B}$ \\
\hline M15 & $1,95 \mathrm{C}$ & $1,71 \mathrm{C}$ \\
\hline M7 & $1,24 \mathrm{D}$ & $1,24 \mathrm{C}$ \\
\hline
\end{tabular}

*Letras iguais na mesma coluna indicam médias equivalentes; **nível de significância 0,05

Fazenda A - Jataí (GO); Fazenda B - Orizona (GO)

\section{Referências}

BERGSTEN, C. Infectious diseases of the digit. In: GREENOUGH, P. R.; WEAVER, D. A. Lameness in cattle. 3. ed. Philadelphia: Saunders, p. 96-100. 1997.

BRITT. J. S.; GASKA, J.; GARRET, E. F.; KONKLE, D.; MEALY, M. Comparison of topical application of three products for treatment of papillomatous digital dermatitis in dairy cattle. Journal American Veterinary Medical Association, Shamburg, n. 209, p. 1134-1136, 1996. CORDEIRO, G. M. Modelos lineares generalizados. Campinas, VII SINAPE, 1986. $286 \mathrm{p}$. a maioria dos animais portadores de enfermidades digitais.

Avaliando-se a propriedade $B$, verificou-se o mesmo comportamento dos escores de cicatrização nos diferentes momentos de avaliação. Porém, aos 45 dias do pósoperatório, houve uma melhor resposta ao tratamento II em relação ao tratamento I, mas ocorrendo o inverso logo em seguida. Essa variação pode ser atribuída a uma melhor condução do pós-operatório nessa propriedade à ocasião.

Os custos dos protocolos terapêuticos empregados no tratamento dos animais alocados no grupo Gl foi estimado em $R \$ 158,96$, no Gll em $R \$, 123,70$ e no Glll em $R \$ 118,99$, ou $\bigcup \$ 69,41, \cup \$ 54,02, \cup \$ 51,96$, respectivamente. Os itens que mais oneraram o tratamento foram a mão-de-obra e a construção do pedilúvio. Segundo Politiek et al. (1986), os problemas com cascos possuem importantes implicações econômicas diretas, tais como gastos com toalete, pedilúvio, mão-de-obra e tratamento veterinário e indiretas, como queda na produção de leite, descarte involuntário, diminuição de fertilidade, da vida útil e do valor do animal para abate.

\section{Conclusão}

No tratamento da dermatite digital bovina em sua fase inicial, o protocolo terapêutico empregado nos animais do grupo Gl, incluindo a curetagem das lesões, toalete dos estojos córneos comprometidos e saudáveis, uso tópico de anti-séptico e antibiótico associados à antibioticoterapia parenteral, apresentou maior eficácia durante os primeiros 45 dias do pós-operatório; contudo, o custo deste protocolo foi superior aos demais.

CUNHA, P. H. J. Pedilúvio para bovinos: avaliação físico-química, microbiológica e eficácia terapêutica das soluções desinfetantes. 2000, 131f. Dissertação (Mestrado em Medicina Veterinária) - Escola de Veterinária, Universidade Federal de Goiás, Goiânia.

DIAS, R. S.; MARQUES JÚNIOR., A. P. Atlas-Casco em bovinos, 2. ed. São Paulo: Lemos Editorial, 2003.67 p.

DIRKSEN, G. Sistema locomotor. In: DIRKSEN, G.; GRÜNDER, H. D.; STÖBER, ROSENBERGER, M. Exame clínico dos bovinos. 3. ed., Rio de Janeiro: Guanabara Koogan, p. 315-340, 1993.

FAJT, V. R.; APLEY, M. D. Antimicrobial tissues in bovine lameness. Veterinary Clinics of North America: Food Animal Practice, Philadelphia, v. 17, n. 1, p. 159-174, 2001. 
FERREIRA, P. M. Enfermidades podais em rebanho leiteiro confinado. 2003, 79 f. Tese (Doutorado em Medicina Veterinária) - Escola de Veterinária, Universidade Federal de Minas Gerais, Belo Horizonte.

GREENOUG, P. R. Diseases of the feet of dairy cows - Infectious diseases of the interdigital space. In: CONGRESSO BRASILEIRO DE CIRÚRGIA E ANESTESIOLOGIA VETERINÁRIA, 4., Goiânia. Anais eletrônicos... [CD-ROM], Goiânia: Temma, 2000.

GREENOUGH, P. R. Cascos irregulares são sinais de problema. Revista Balde Branco, São Paulo, n. 388, p. 9-13, 1997.

GREENOUGH, P. R.; WEAVER, A . D. Lameness in cattle. 3. ed., Philadelphia, W. B. Saunders, 1997, 336 p.

KAMILOGLO, A.; DEMIRKAN, I.; BARAN, V. Comparisson of ceftiofur sodium by intravenous region antibiotherapy and local oxtetracycline application for treatment of bovine digital dermatitis. Kafkas Universitesi Veteriner Dergise, n. 8, v. 2, p. 107-110, 2002.

LUGINBUHL A., KOLLBRUNNER, M. Increasing number of cattle suffering from papillomatous digital dermatitis in Switzerland. Schweizer Archiv fur Tierheilkunde, v. 142, p. 513-519, 2000.

MANSKE, T., HULTGEEN, J., BERGSTEN, C. Topical treatment of digital dermatitis associated with severe heel-horn erosion in a Swedish dairy herd. Preventive Veterinary Medicine, Amsterdan, v. 53, p. 215 231, 2002.

MAREGA, L. M. Ocorrência e tratamento de lesões podais semelhantes à dermatite digital em bovinos. 2001. 72 f. Dissertação (Mestrado) Faculdade de Ciências Agrárias e Veterinárias -UNESP - Jaboticabal, SP.

MASSONE, F. Miorelaxantes. In: Anestesiologia veterinária. farmacologia e técnicas. 4. ed. Rio de Janeiro: Guanabara Koogan, cap. 9, p. 94-102, 2003.

NICOLETTI, J. L. M. Manual de podologia bovina. Barueri: Manole, 2004, $125 \mathrm{p}$.
POLITIEK, M. J.; DISTL, O.; FJELDAAS, T. Importance of claw quality in cattle: review and recomendations to achieve genetic improviment. Livestock Production Science, Livestock, v. 115, p. 133-152, 1986.

QUINN, P. J.; CARTER, G. R. Clinical veterinary microbiology, London: Wolf, 1994.648 p.

RUIZ, R. L. Microbiologia zootécnica. São Paulo: Roca, 1992. 314 p. SAS â. 1997. User's Guide: Statistics, Version 8th. SAS Institute Inc. Cary, NC.

SHEARER, J. K. Lameness of dairy cattle: Consequence and causes. The Bovine Practioner, Stillwater, v. 1, n. 32, p. 79-85, 1998.

SILVA, L. A. F.; ATAYDE, I. B.; FIORAVANTI, M. C. S.; EURIDES, D.; OLIVEIRA, K. S.; SILVA, C. A.;VIEIRA, D.; ARAÚJO, E. G. Comparative study of three surgical treatments for two forms of the clinical presentation of bovine pododermatitis. Annals of the New York Academy of Sciences, New York, v. 1026, p. 118-124, 2004.

SILVA, L. A. F.; SILVA, L. M.;ROMANI, A. F;RABELO, R. E;FIORAVANTI, M. C. S.; SOUZA, T. M.; SILVA, C. A. Características clínicas e epidemiológicas das enfermidades podais em vacas lactantes do município de Orizona-GO. Ciência Animal Brasileira, Goiânia, v. 2, n. 2, p. 119-126, 2001a.

SILVA, L. A. F.; SILVA, L. M.; ROMANI, A. F.; RABELO, R. E.; FIORAVANTI, M. C. S.;BORGES, G. T.; VERÍSSIMO, A. C. C. Uma proposta de tratamento para afecções podais em bovinos. In: CONGRESSO BRASILEIRO DE BUIATRIA, 4., 2001b, Campo Grande. Anais... Campo Grande, 2001b, [CDROOM].

SILVA, L. A. F.; SILVA, C. A.; BORGES, J. R. J.; FIORAVANTI, M. C. S.; BORGES, G. T.; ATAYDE, I. B. A clinical trial to asses the use of sodium hypochlorite and oxytetracycline on the healing of digital dermatitis lesions in cattle. Canadian Veterinary Journal, Ottawa, v. 46, n. 4, 2005. 